D. MARINER RESULTS 


\title{
MARINER 6: ULTRAVIOLET SPECTRUM OF MARS UPPER ATMOSPHERE*
}

\author{
C. A. BARTH \\ Dept. of Astro-Geophysics and Laboratory for Atmospheric and Space Physics, University of \\ Colorado, Boulder, Colo., U.S.A. \\ W. G. FASTIE \\ Dept. of Physics, Johns Hopkins University, Baltimore, Md., U.S.A. \\ C. W. HORD, J. B. PEARCE, K. K. KELLY, A. I. STEWART, \\ G. E. THOMAS, and G. P. ANDERSON \\ Dept. of Astro-Geophysics and Laboratory for Atmospheric and Space Physics, University of \\ Colorado, Boulder, Colo., U.S.A. \\ and \\ O. F. RAPER \\ Space Sciences Division, Jet Propulsion Laboratory, Pasadena, Calif., U.S.A.
}

\begin{abstract}
Emission features from ionized carbon dioxide and carbon monoxide were measured in the 1900- to $4300-\AA$ spectral region. The Lyman- $\alpha 1216-\AA \AA$ line of atomic hydrogen and the 1304-, $1356-$, and $2972-\AA$ lines of atomic oxygen were observed.
\end{abstract}

The flight of Mariner 6 past Mars on 31 July 1969 presented the first opportunity to measure the ultraviolet dayglow of that planet. The technique of using ultraviolet spectroscopy to study planetary atmospheres has been developed both theoretically and experimentally over the past $9 \mathrm{yr}$ [1]. Rocket experiments have shown that the ultraviolet dayglow of the earth consists of the following emission features: the Lyman- $\alpha$ $1216-\AA$ line of atomic hydrogen; the 1304-, 1356-, and 2972- $\AA$ lines of atomic oxygen; the 1200-, 1493-, 1744-, and 3466- $\AA$ lines of atomic nitrogen; the LymanBirge-Hopfield, Vegard-Kaplan, and second-positive bands of molecular nitrogen; the gamma bands of nitric oxide; and the first negative bands of ionized molecular nitrogen [2]. These emissions are produced in the earth's upper atmosphere by resonance and fluorescence scattering of ultraviolet solar radiation and by photoelectron impact excitation.

The Mariner ultraviolet spectrometer was specifically designed to measure emissions from the sunlit atmosphere above the limb of Mars. Extensive baffling in front of the telescope suppressed off-axis light from entering the 250-mm Ebert-Fastie spectrometer [1]. Two photomultiplier tubes simultaneously recorded the spectral

* The success of this experiment, which has been under preparation for $9 \mathrm{yr}$, is the result of the efforts of a large number of people at NASA headquarters, the Jet Propulsion Laboratory, the University of Colorado, Johns Hopkins University, and elsewhere in the scientific community. The large scientific return from the Mariner 1969 mission is due to the technical and managerial skills of H. M. Schurmeier and the Mariner project staff at JPL and NASA headquarters. Supported by NASA under JPL contract 951790 and NASA grant NGL 06-003-052. Reprinted from Science 165, 1004-1005.

Sagan et al. (eds.), Planetary Atmospheres, 253-256.

All Rights Reseried. Copyright C 1971 by the I.A.C. 
scans which occurred repetitively every $3 \mathrm{sec}$. The wavelength band at 1100 to $1900 \AA$ was measured at a resolution $10 \AA$ by a cesium iodide tube, and the 1900 - to $4300-\AA$ band was measured at a resolution of $20 \AA$ with a bi-alkali tube.

The first observation of the sunlit atmosphere of Mars occurred when Mariner 6 was $8300 \mathrm{~km}$ from the planet's center, the slant range to the limb was $7600 \mathrm{~km}$, and the solar zenith angle at the limb was $27^{\circ}$. The telescope baffling rejected the off-axis light from the disc sufficiently well so that a spectrum rich in emission features was obtained. In the spectral interval from 1900 to $4300 \AA$, the bi-alkali photomultiplier tube recorded the spectrum shown in Figure 1.

An initial spectroscopic analysis has been performed with the use of laboratory and theoretical data prepared before the Mars encounter. A comparison spectrum synthesized from three separate sources is shown in Figure 2 together with the individual spectra. Figure $2 \mathrm{a}$ was obtained from a spectrum of the Martian disc measured later in the flight by the Mariner spectrometer. This spectrum is the result of Rayleigh

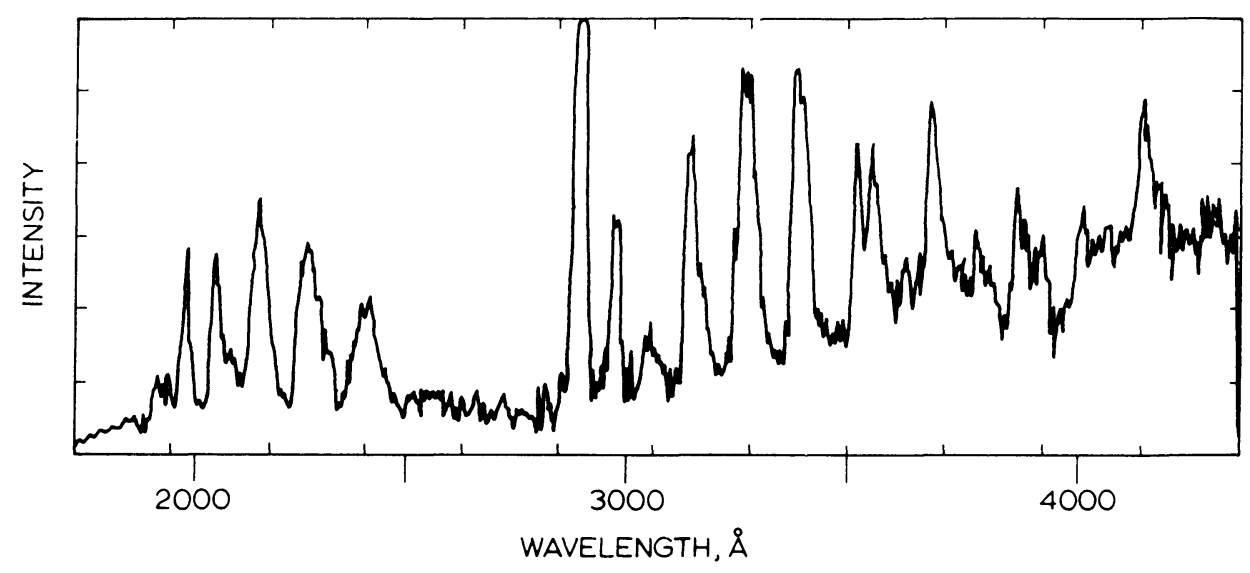

Fig. 1. Ultraviolet spectrum of Mars upper atmosphere. A nominal wavelength scale is shown on the abscissa. The ordinate is an arbitrary intensity scale uncorrected for the spectral response of the instrument. A number of spurious noise pulses have been edited out of this spectrogram.

scattering and ground reflection of solar radiation. The spectrum in Figure $2 b$ was produced in the laboratory by the bombardment of carbon dioxide at $10^{-3}$ torr by electrons with an energy of $20 \mathrm{eV}$. This spectrum contains the prominent ionized carbon dioxide emission feature at $2890 \AA$ and the Fox-Duffendack-Barker bands. The spectrum in Figure $2 \mathrm{c}$ was composed theoretically from the calculations of how the Cameron bands of carbon monoxide appear in the fluorescence scattering of sunlight [2], and it has been adjusted for the response of the instrument. The three spectra were normalized individually and summed to form the composite spectrum in Figure $2 \mathrm{~d}$. In view of the way in which we put together the synthetic spectrum in Figure 2d, the similarity between it and the Mars spectrum in Figure 1 is remarkable. However, the excitation mechanisms which occur in the upper atmosphere of Mars may or may not be the ones we used to produce the synthetic spectrum. 


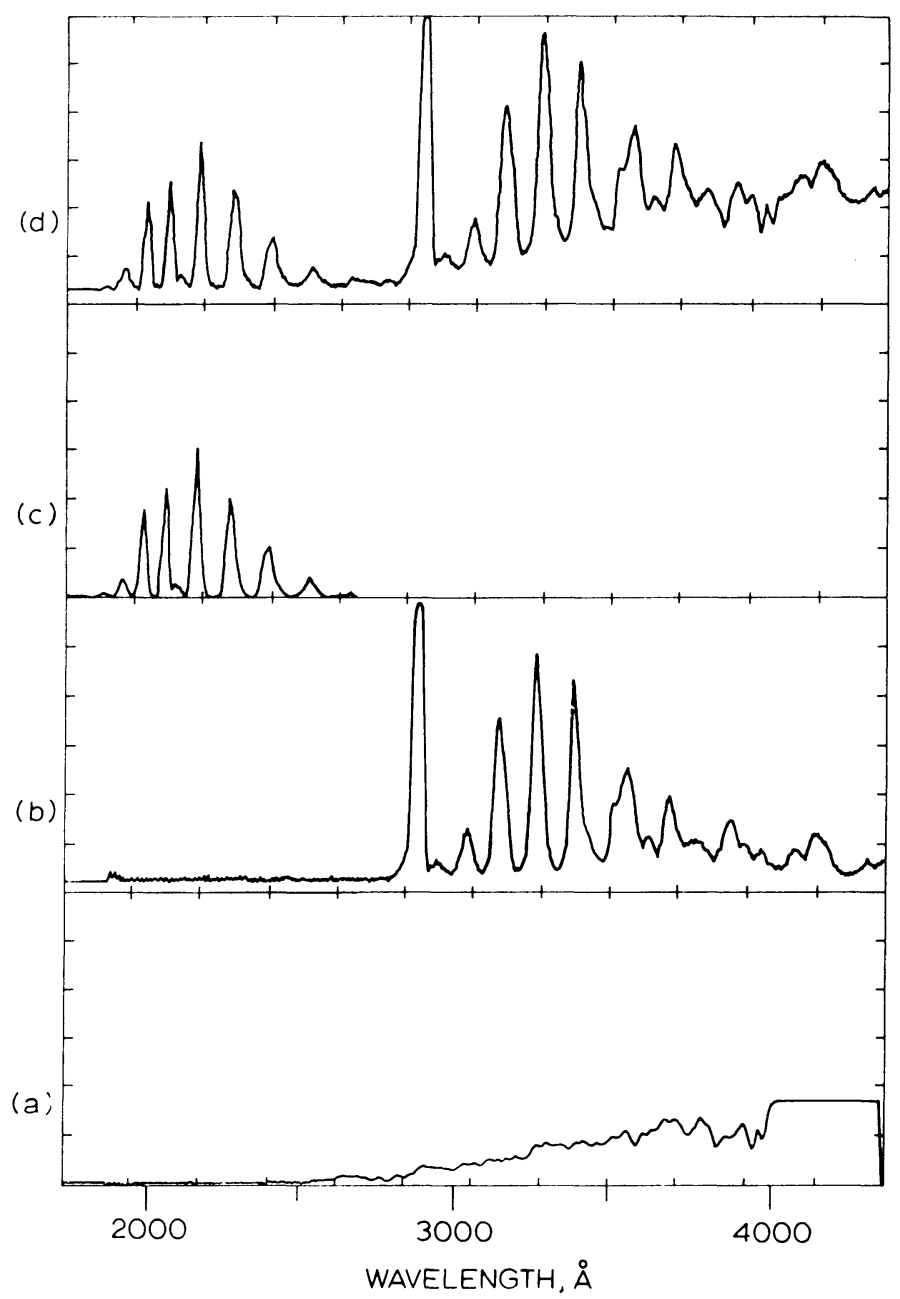

Fig. 2. Synthesis of comparison spectrum. (a) Off-axis light component from Mars disc. (b) Carbon dioxide spectrum from laboratory electron impact experiment. (c) Carbon monoxide spectrum from theoretical calculation of fluorescence scattering. (d) Composite spectrum synthesized by adding $\mathrm{a}, \mathrm{b}$, and $\mathrm{c}$.

We have identified an additional feature which appears in the Mars spectrum as the $2972-\AA$ line of atomic oxygen. There may be further unidentified features in this spectrum.

The limb spectrum in the 1100 - to 1900 - $\AA$ region was recorded by the cesium iodide photomultiplier tube. The principal emission features that were observed were: the Lyman- $\alpha 1216-\AA$ line of atomic hydrogen, the 1304- and $1356-\AA$ lines of atomic oxygen, and the fourth-positive bands of carbon monoxide.

One particularly important objective of the ultraviolet spectrometer experiment was to search for nitrogen in the atmosphere of Mars. This first analysis shows no evidence 18 -P.A. 
of nitrogen emissions in the ultraviolet spectrum of the upper atmosphere. The following emissions were searched for and found missing: second-positive and LymanBirge-Hopfield bands of molecular nitrogen, first-negative bands of ionized molecular nitrogen, gamma bands of nitric oxide, and 1200- and 1493- $\AA$ lines of atomic nitrogen. The final analysis of these data will allow an upper limit to be placed on the amount of nitrogen in the upper atmosphere of Mars.

Repetitive spectra were taken as the Mariner spectrometer crossed the limb of Mars. These data, which contain information about the scale height of individual spectral emissions will be used to construct a model of the Mars upper atmosphere. This first report is simply a record of our identifications in the ultraviolet spectrum of the upper atmosphere. The instrument also obtained spectra of the bright and dark parts of the disc, the terminator, and the atomic hydrogen corona.

\section{References}

[1] Barth, C. A.: 1969, Appl. Opt. 8, 1295.

[2] Barth, C. A.: 1966, in The Middle Ultraviolet - Its Science and Technology (ed. by A. E. S. Green), Wiley, New York; Fastie, W. G., Crosswhite, H. M., and Heath, D. F.: 1964, J. Geophys. Res. 69, 4129; Barth, C. A.: 1964, J. Geophys. Res. 69, 3301; Barth, C. A. and Pearce, J. B.: 1966, Space Res. 6, 381; unpublished results from a rocket flight, 13 June 1969. 